\title{
Quantum associative memory with linear and non-linear algorithms for the diagnosis of some tropical diseases
}

\author{
J-P. TCHAPET NJAFA, S.G. NANA ENGO \\ Laboratory of Photonics, Department of Physics, \\ University of Ngaoundere, PO. BOX 454 Ngaoundere, Cameroon
}

September 25, 2018

\begin{abstract}
This paper presents the QAMDiagnos, a model of Quantum Associative Memory (QAM) that can be a helpful tool for medical staff without experience or laboratory facilities, for the diagnosis of four tropical diseases (malaria, typhoid fever, yellow fever and dengue) which have several similar signs and symptoms. The memory can distinguish a single infection from a polyinfection. Our model is a combination of the improved versions of the original linear quantum retrieving algorithm proposed by Ventura and the non-linear quantum search algorithm of Abrams and Lloyd. From the given simulation results, it appears that the efficiency of recognition is good when particular signs and symptoms of a disease are inserted given that the linear algorithm is the main algorithm. The non-linear algorithm helps confirm or correct the diagnosis or give some advice to the medical staff for the treatment. So, our QAMDiagnos that has a friendly graphical user interface for desktop and smart-phone is a sensitive and a low-cost diagnostic tool that enables rapid and accurate diagnosis of four tropical diseases.
\end{abstract}

\section{Introduction}

Diagnosis is the identification of a situation, a difficulty or a phenomenon by interpreting external signs or lesions. In the medical field, it consists of identifying abnormal condition that afflicts a specific patient, based on manifested clinical data. If the final diagnosis matches a disease that afflicts a patient, the diagnostic process is correct; otherwise, a misdiagnosis occurs. Medical diagnosis can also be defined as the process that allows a physician, through information collected from a history and physical examination of a person, to make prediction about features of clinical situations and determine appropriate course of action. It implements a complex decision process that involves a lot of vagueness and uncertainty management, especially when a disease has multiple signs and symptoms or non-specific signs and symptoms.

From the point of view of statistics, the diagnostic procedure involves classification tests. That is, the task is made on the basis of measured features to assign a patient to one of a small set of classes [1]. Then, Artificial Neural Networks (ANN) provide powerful tools that help physicians avoid misdiagnosis by analyzing, modelling and making sense of complex clinical data across a broad-range of medical applications. As ANNs have the ability of prediction, parallel operation and adaptability, they have been widely used as computer-assisted tools among many techniques about automatic disease diagnosis proposed in the literature $[2,3]$. ANNs have been used for example for the diagnosis of colorectal cancer, multiple sclerosis lesions, colon cancer, tuberculosis, pancreatic disease, gynecological diseases and early diabetes [2].

Associative Memories (AM) are a class of ANNs specialised in pattern recognition that have drawn the attention of major research groups in the world due to a number of properties such as a rapidity, a compute efficient best-match and an intrinsic noise tolerance. Aldape-Pérez et al. gave in the ref. [4] a good summary of the AM evolution, from the first model, the Lernmatrix developed by Karl Steinbuch in 1961, to the recent model proposed between 1982-1984 by Hopfield $[5,6]$. The latter is an AM that uses the Hebbian learning rule and it is able to recall patterns from noisy or partial representation.

Some computer-assisted tools in the case of tropical diseases, are already built and some of them use ANNs, but they are mainly specialized for malaria $[7,8,9,10,2]$. It is worth noting that malaria is the most parasitic disease spread over the world. $40 \%$ of the world's population are concerned, especially those of tropical regions. In Cameroon, as in the most Sub-Saharan countries, it is a public health problem because the whole population is exposed to the disease. To diagnose malaria, the World Health Organisation (WHO) [11] recommends the use of rapid diagnostic tests. However these tools need some conservation facilities that are difficult to find in rural and semi-urban regions in developing countries. So, the most widely used technique for determining the development stage of malaria is through visual microscopical evaluation of Giemsa stained blood smears. However, this is 
a routine and time-consuming task and it requires a well-trained operator. In addition, there is frequently a misdiagnosis because of confusion between signs and symptoms of malaria and that of other tropical diseases such as typhoid fever, yellow fever and dengue, or inexperience of medical staff. As malaria, these tropical diseases are also life-threatening.

In order to provide a rapid and accurate tool for the diagnosis of the above mentioned four tropical diseases (malaria, typhoid fever, yellow fever and dengue), we propose the framwork QAMDiagnos, Quantum Associative Memory for the Diagnosis, associated with a friendly multi-platform graphical user interface (Android, Linux, MS Windows). It is a combination of improved versions of the original linear quantum retrieving algorithm proposed by Ventura [12] and the non-linear quantum search algorithm of Abrams and Lloyd [13]. The aim of QAMDiagnos is to (i) act as an advisory tool to inexperienced medical staff, especially senior nurses in rural health centres having no or a limited number of physicians; (ii) act as a decision-support tool for medical diagnosis for physicians in under-staffed health centres; (iii) provide an alternative way to reach a reasonable tentative diagnosis, and hence early commencement of clinical management of patients in the absence of laboratory facilities in many rural and semi-urban health centres; (iv) facilitate the treatment and prevent potential pandemics given the fact that today the increase of international air travel/traffic, tourism to tropical regions and human migration led to a rising incidence of tropical diseases (since vaccines are unavailable for most major tropical infections). Our framework, which is more robust than that of Agarkar and Ghathol [14] that uses the FFANN for the diagnosis of malaria, typhoid fever and dengue, can be extended to a wide range of tropical diseases.

The paper is structured as it follows: Section 2 provides a description of foundations of our model of Quantum Associative Memory. In Section 3 a brief description of each disease is given. Signs and symptoms of diseases are given in Appendix A. Section 4 is devoted to simulations results and discussion whereas in Section 5 we explain the use of the multi-platform friendly graphical user interface (GUI) of QAMDiagnos, designed for the medical staff. Finally, we conclude with an outlook of possible future improvements.

\section{Description of Quantum Associative Memory}

Associative Memories are a class of Artificial Neural Networks that can memorise information, field of knowledge or patterns and can retrieve that from partial or noisy data. Quantum Associative Memories (QAM) combine neurocomputing with quantum computations. Therefore, QAM models share main features both of quantum information theory and Associative Memory.

\subsection{Few basic concepts of quantum information theory}

\subsubsection{Quantum bit}

Quantum information use a quantum bit or a qubit instead of an ordinary bit as the fundamental unit of information. The qubit can be an atom, a molecule or a photon that can be in a superposed state (energy or spin for example). As the classical bit, the qubit can take two particular states noted $|0\rangle$ and $|1\rangle$ that are the basis states of a Hilbert space $\mathcal{H}$ of 2 dimensions. The fundamental difference between the qubit and the classical bit is that the qubit can take simultaneously both values. So, the state of the qubit can be represented by the following superposed state:

$$
|\psi\rangle=\alpha|0\rangle+\beta|1\rangle
$$

with at any time

$$
|\alpha|^{2}+|\beta|^{2}=1, \quad \alpha, \beta \in \mathbb{C} .
$$

$|\alpha|^{2}$ and $|\beta|^{2}$ are the probabilities of the qubit to be found in the states $|0\rangle$ and $|1\rangle$ respectively after a measurement. $(|0\rangle,|1\rangle)$ is the most used computational basis that is a pair of orthonormal vectors defined as

$$
|0\rangle=\left(\begin{array}{l}
1 \\
0
\end{array}\right), \quad|1\rangle=\left(\begin{array}{l}
0 \\
1
\end{array}\right)
$$

Therefore, quantum superposition suggest that an $n$-qubits register can exist in all its possible $2^{n}$ states at the same time.

\subsubsection{Quantum entanglement}

The use of only one qubit does not highlight the power of quantum information. A purely quantum phenomenon that has non classical analogue is a quantum entanglement. We are talking about the quantum entanglement when at least a pair of qubits has quantum correlation. It means that any interaction with one of the qubits affects instantaneously the other despite the separation distance, highlighting the non-local features of quantum theory. In this case, we should see the whole qubits as one unique physical system instead of many separated 
subsystems. So, no classical consideration can allow to know the state of each qubit. A well-known entangled states are the following EPR states:

$$
\left|\Psi^{+}\right\rangle=\frac{1}{\sqrt{2}}(|00\rangle+|11\rangle),\left|\Psi^{-}\right\rangle=\frac{1}{\sqrt{2}}(|00\rangle-|11\rangle),\left|\Phi^{+}\right\rangle=\frac{1}{\sqrt{2}}(|01\rangle+|10\rangle),\left|\Phi^{-}\right\rangle=\frac{1}{\sqrt{2}}(|01\rangle-|10\rangle),
$$

that cannot be written as the products of independent states of two separate subsystems.

\subsubsection{Quantum parallelism and decoherence}

Due to the quantum superposition and the quantum entanglement it is possible to perform multiple computations simultaneously or quantum parallelism on a system. But a measurement on the system destroys the quantum superposition and the system collapses to one of its possible states. This phenomenon is the quantum decoherence that can be seen as an interaction between a qubit and its environment. One of the challenges of quantum processing consists of increasing the probability to observe a needed state before the quantum decoherence occurs.

\subsubsection{Elementary quantum gate}

Quantum computing (that is changing the state of a qubit to another) is carried out through unitary operators or quantum logic gates when they are used in quantum circuit. Unlike many classical logic gates, the quantum logic gates are reversible, and therefore allow to avoid energy dissipation. Let us recall that the operator $\mathrm{U}$ is an unitary operator if

$$
\mathrm{UU}^{\dagger}=\mathrm{U}^{\dagger} \mathrm{U}=\mathbb{I},
$$

where $\mathbb{I}$ is the identity operator, and $U^{\dagger}$ the complex conjugate transpose of U. Any unitary operator can be written as

$$
\mathrm{U}=\exp (-i \mathbf{G})
$$

where $\mathrm{G}$ is an hermitian operator, that is $\mathrm{G}=\mathrm{G}^{\dagger}$.

In the present work, the NOT gate $\mathrm{X}=|1-x\rangle\langle x|$ and the Walsh-Hadamard gate $\mathrm{W}=\frac{1}{\sqrt{2}}\left((-1)^{x}|x\rangle\langle x|+| 1-x\rangle\langle x|\right)$ will be the most using single-qubit quantum logic gates:

$$
\begin{gathered}
|x\rangle-\mathrm{X}-|1-x\rangle \quad \mathrm{X}=\left(\begin{array}{cc}
0 & 1 \\
1 & 0
\end{array}\right) \\
|x\rangle-W-\frac{1}{\sqrt{2}}\left((-1)^{x}|x\rangle+|1-x\rangle\right) \quad \mathrm{W}=\frac{1}{\sqrt{2}}\left(\begin{array}{cc}
1 & 1 \\
1 & -1
\end{array}\right),
\end{gathered}
$$

where $x \in\{0,1\}$.

The two-qubit quantum logic gate mostly used in this work will be the controlled NOT gate $\mathrm{CX}=(|0\rangle\langle 0|) \otimes$ $\mathbb{I}+(|1\rangle\langle 1|) \otimes \mathrm{X}:$

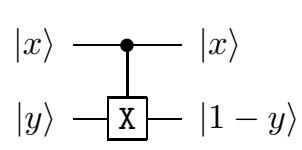

$$
\mathrm{CX}=\left(\begin{array}{ll}
\mathbb{I} & \mathbb{O} \\
\mathbb{O} & \mathrm{X}
\end{array}\right)=\left(\begin{array}{ll|ll}
1 & 0 & 0 & 0 \\
0 & 1 & 0 & 0 \\
\hline 0 & 0 & 0 & 1 \\
0 & 0 & 1 & 0
\end{array}\right)
$$

where $x, y \in\{0,1\}$. The $\mathrm{CX}$ gate acts on two-qubits and performs the $\mathrm{X}$ operation on the second qubit only when the first qubit is in the state $|1\rangle$, and otherwise leaves it unchanged.

\subsection{Few basic concepts of Associative Memories}

A Neural Network, more properly referred to as an Artificial Neural Network (ANN), is a computing system made up of several important basic elements, which include the concept of a processing element (neuron), the transformation performed by this element (in general, input summation and non-linear mapping of the result into an output value), the interconnection structure between neurons, the network dynamics, and the learning rules that govern the modification of interconnection strengths [15]. A major dichotomisation of Neural Networks can be realised by considering whether they are trained in a supervised or unsupervised manner. An example of the latter is the Hopfield model of content-addressable memory, or Associative Memory, using the concept of attractor states. This model has an apparent similarity to human episodic memory: it can recall patterns after a single exposure using a Hebbian learning rule, and it is capable to retrieve the above mentioned patterns from partial information, partial and noisy information or noisy ones.

In short, Associative Memories are Neural Networks centralised around two algorithms. The first is to memorise information or patterns and known as learning algorithm. The second is for the restitution of learned information or patterns from partial or noisy data. It is known as retrieving algorithm. 
Related to quantum theory, Associative Memories are called Quantum Associative Memories (QAM) where the learning and retrieving algorithms are quantum algorithms. The QAM is one of the most promising approaches to quantum neurocomputing. The linear part of the QAM design here is an improved version of the one built by Ventura and Martinez where the stored patterns are considered as the basis states of the memory quantum state $[16]$.

It should be noted that in the classical Hopfield network the existence of symmetric, Hebbian connections, guarantees the stability of a unique stored pattern; similarly, in a quantum analogue of the Hopfield network the quantum entanglement ensures the integrity of a stored pattern (basis state).

The Tab. 1 summarises the analogies used in developing a QAM [15].

\begin{tabular}{ll}
\hline Classical Neural Networks & Quantum Associative Memory \\
\hline Neuronal state $x_{i} \in\{0,1\}$ & Qubit $|x\rangle=\alpha|0\rangle+\beta|1\rangle$ \\
Connections $\left\{w_{i j}\right\}_{i j=1}^{p-1}$ & Quantum entanglement $\left|x_{0} x_{1} \ldots x_{p-1}\right\rangle$ \\
Learning rule $\sum_{s=1}^{p} x_{i}^{s} x_{j}^{s}$ & Superposition of entangled states $\sum_{s=1}^{p} \alpha_{s}\left|x_{0} x_{1} \ldots x_{p-1}\right\rangle$ \\
Winner search $n=\max _{i} \arg \left(f_{i}\right)$ & Unitary transformation $\mathrm{U}|\psi\rangle=\left|\psi^{\prime}\right\rangle$ \\
Output result $n$ & Decoherence $\sum_{s=1} \alpha_{s}\left|x^{s}\right\rangle \Rightarrow\left|x^{k}\right\rangle$ \\
\hline
\end{tabular}

Table 1: Corresponding concepts from the domains of classical Neural Networks and Quantum Associative Memory.

It is worth noting that there are some other interesting models of quantum inspired neural networks and quantum inspired evolutionary optimization algorithms [17, 18, 19, 20].

In Subsections 2.3 and 2.4 we will briefly describe the quantum learning and quantum retrieving algorithms used on our QAM model. The full details can be found in refs. [12] and [13].

\subsection{Quantum learning algorithm}

For our QAM an operator name BDD is used as the learning algorithm. The BDD operator is obtained by using the Binary Superposed Quantum Decision Diagram (BSQDD) proposed by Rosenbaum [21]. Contrarily to other quantum learning algorithms such as the one of Ventura [22], which needs the initial state to be $|00 \ldots 0\rangle$, the BSQDD is computed by using any basis states $|z\rangle$ of the Hilbert space of $2^{n}$ dimensions. The idea behind the BSQDD is to represent a quantum superposition as a decision diagram where each node corresponds to a gate. The gate that corresponds to the node on each branch of the BSQDD is controlled by the path used to reach it from the root of the decision diagram. Thereby three steps, given by Algorithm 1, are needed to construct one BSQDD.

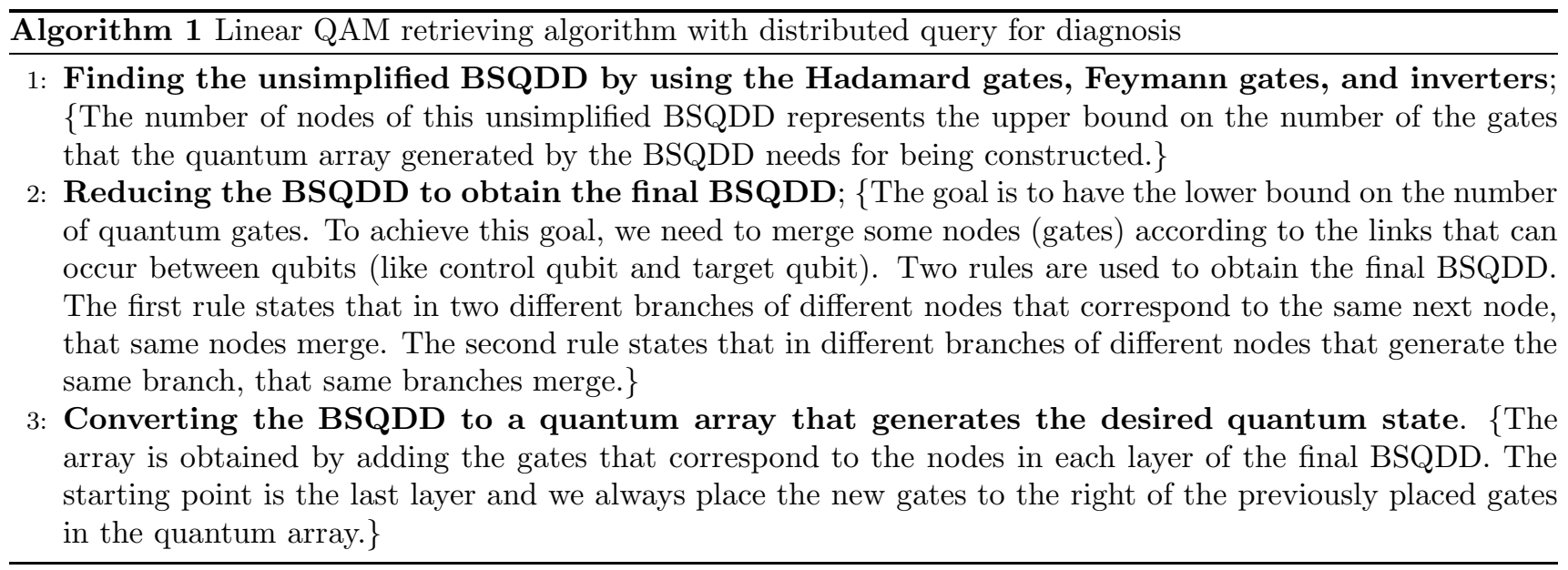

Example 1 Fig. 1 gives the three steps allowing to construct the state $\sqrt{\frac{1}{5}}(|000\rangle+|010\rangle+|110\rangle+|001\rangle+|101\rangle)$ from the starting state $|000\rangle$. The elementary gates used are respectively the rotation gates

$$
\mathrm{R}(\theta)=\left(\begin{array}{cc}
\sqrt{\frac{3}{5}} & \sqrt{\frac{2}{5}} \\
\sqrt{\frac{2}{5}} & -\sqrt{\frac{3}{5}}
\end{array}\right), \mathrm{R}(\alpha)=\left(\begin{array}{cc}
\sqrt{\frac{2}{3}} & \frac{1}{\sqrt{3}} \\
\frac{1}{\sqrt{3}} & -\sqrt{\frac{2}{3}}
\end{array}\right),
$$


the Hadamard gate $\mathrm{W}$, and the NOT gate $\mathrm{X}$. Then $\left|\psi_{3}\right\rangle=\sqrt{\frac{3}{5}}|000\rangle+\sqrt{\frac{2}{5}}|100\rangle$ and $\left|\psi_{2}\right\rangle=\sqrt{\frac{2}{5}}|000\rangle+\frac{1}{\sqrt{5}}|010\rangle+$ $\frac{1}{\sqrt{5}}|100\rangle+\frac{1}{\sqrt{5}}|110\rangle$.

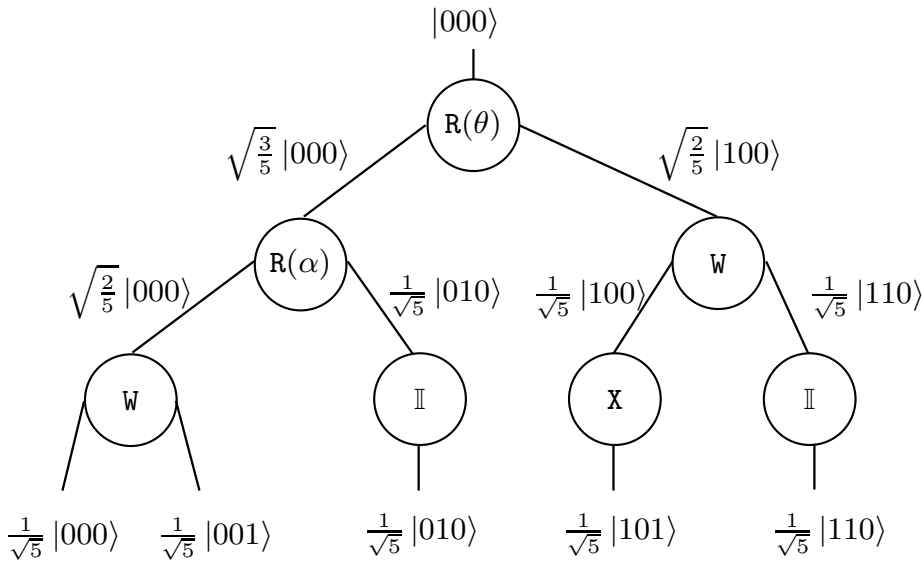

(a) Unsimplified BSQDD

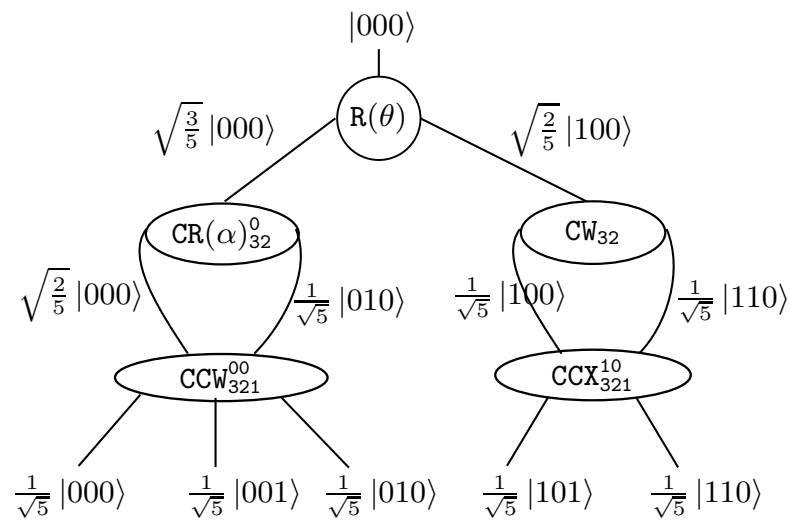

(b) Final BSQDD

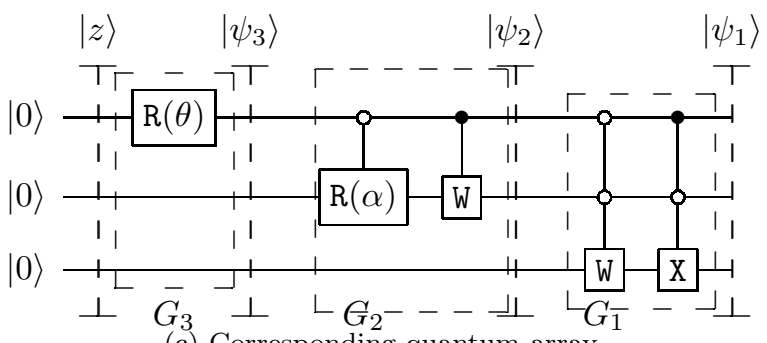

(c) Corresponding quantum array

Figure 1: BSQDD to obtain the state $\left|\psi_{1}\right\rangle=\sqrt{\frac{1}{5}}(|000\rangle+|010\rangle+|110\rangle+|001\rangle+|101\rangle)$. The two BSQDDs of the Fig. are equivalent.

\subsection{Quantum retrieving algorithms}

We use two quantum retrieving algorithms that work together but with different learning approaches.

\subsubsection{Linear QAM algorithm}

The linear QAM retrieving algorithm given by Algorithm 2 is a slightly modified version of QAM-C1 that we have proposed in ref. [12]. It uses an exclusion learning approach. That approach states that the system must be in the superposition of all the possible states, except the pattern states. Let us consider $M$ as a set of patterns and $m$ the number of patterns of length $n$, then

$$
|\Psi\rangle=\frac{1}{\sqrt{N-m}} \sum_{x \notin M}^{N-1}|x\rangle, N=2^{n} .
$$

In the Algorithm 2,

- $\mathcal{O}$ is the oracle operator that inverts the phase of the query state $\left|R e q^{p}\right\rangle$,

$$
\begin{aligned}
& \mathcal{O}=\mathbb{I}-\left(1-e^{i \pi}\right)\left|\operatorname{Re} q^{p}\right\rangle\left\langle\operatorname{Re} q^{p}\right| \\
& \mathcal{O}: a_{x} \mapsto a_{x}-2 \operatorname{Re} q_{x}^{p}\left(\sum_{x=0}^{2^{n}-1}\left(\operatorname{Re} q_{x}^{p}\right)^{*} a_{x}\right),
\end{aligned}
$$

where $a_{x}$ is the probability amplitude of the state $|x\rangle$. The distributed query $\left|R e q^{p}\right\rangle$ is in the following superposed states

$$
\left|R e q^{p}\right\rangle=\sum_{x=0}^{N-1} \operatorname{Re} q_{x}^{p}|x\rangle
$$




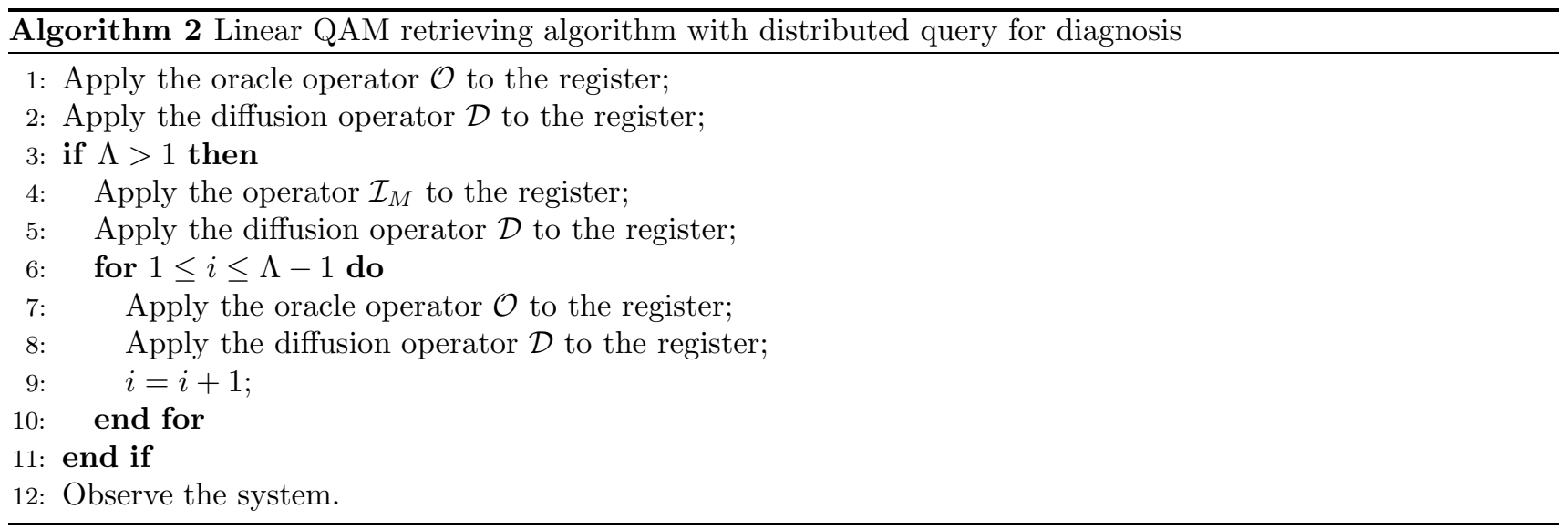

where $R e q_{x}^{p}$ obey to binomial distribution

$$
\left\|R e q_{x}^{p}\right\|^{2}=a^{d_{H}(p, x)}(1-a)^{n-d_{H}(p, x)} .
$$

In equation (14),

- $p$ marks the state $|p\rangle$ that is referred as the query centre;

$-a \in] 0, \frac{1}{2}[$ is an arbitrary value that tunes the width of the distribution;

- the Hamming distance $d_{H}(p, x)=|p-x|$ between binary strings $p$ and $x$ is an important tool that gives the correlation between input and output;

- the amplitudes are such that $\sum_{x}\left\|R e q_{x}^{p}\right\|^{2}=1$.

- $\mathcal{D}$ is the diffusion operator that inverts the probability amplitude of the states of $|\Psi\rangle$ over their average amplitude and for the others over the value 0 :

$$
\begin{aligned}
& \mathcal{D}=\left(1-e^{i \pi}\right)|\Psi\rangle\langle\Psi|-\mathbb{I}, \\
& \mathcal{D}: a_{x} \mapsto 2 m_{x}\left(\sum_{x=0}^{N-1} m_{x}^{*} a_{x}\right)-a_{x},
\end{aligned}
$$

where $m_{x}$ is the probability amplitude of a state of $|\Psi\rangle$.

- $\Lambda$ is the number of iterations that yields the maximal value of amplitudes, which must be as far as possible nearest to an integer,

$$
\Lambda=T\left(\frac{1}{4}+\alpha\right), T=\frac{2 \pi}{\omega}, \alpha \in \mathbb{N}
$$

with the frequency of Grover

$$
\omega=2 \arcsin B, B=\frac{1}{\sqrt{N-m}} \sum_{x=0, x \notin M}^{N-1} R e q_{x}^{p} .
$$

- According to the approach QAM-C1 of the ref. [12] $\mathcal{I}_{M}$ inverts only the phase of the memory pattern states as in the model of Ventura,

$$
\begin{gathered}
\mathcal{I}_{M}=\mathbb{I}-\left(1-e^{i \pi}\right)|\varphi\rangle\langle\varphi|,| \varphi\rangle\left\langle\varphi\left|=\sum_{x \in M}\right| x\right\rangle\langle x|, \\
\mathcal{I}_{M}: a_{x} \mapsto\left\{\begin{array}{l}
-a_{x} \text { if }|x\rangle \in M \\
a_{x} \text { if not. }
\end{array}\right.
\end{gathered}
$$

The linear QAM algorithm is used here as main algorithm given that it increases the probability amplitude of the searched disease. 


\subsubsection{Non-linear QAM algorithm}

The non-linear QAM retrieving algorithm given by Algorithm 3 is formally the same with the one we have proposed in ref. [13] (see that ref. for more details) that is an improved version of the one given by [23]. It uses inclusion learning approach.

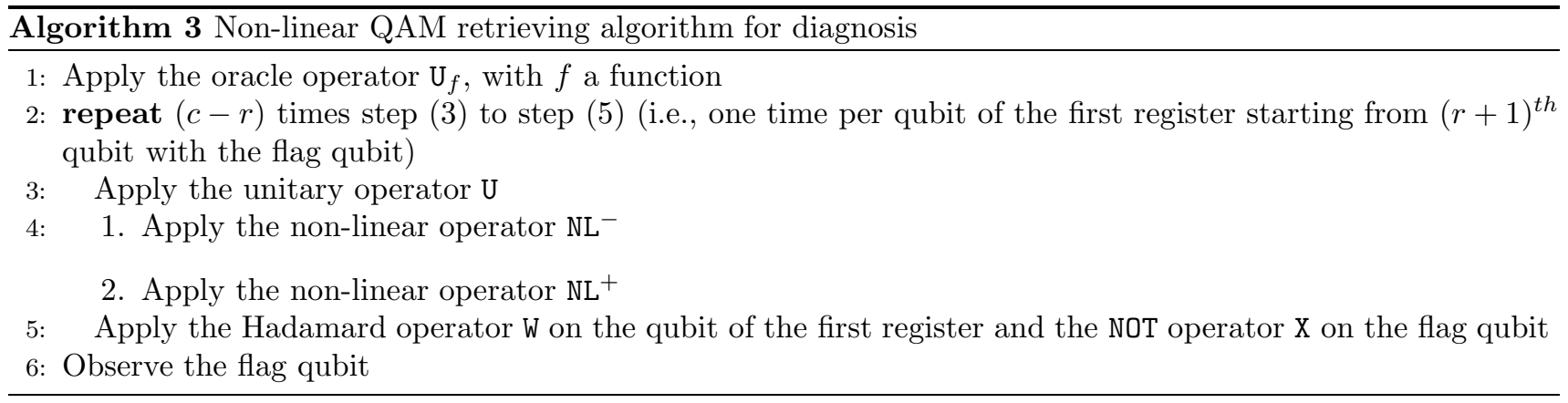

Algorithm 3 is used on the subspace of signs and symptoms and allows to know if signs and symptoms related to a particular disease exist in the database. In the Algorithm 3,

- $n$ is the number of qubits of the first register.

- $p \leq 2^{n}$ is the number of stored patterns.

- $q \leq p$ is the number of stored patterns if the values of $t$ qubits are known (i.e. $t$ qubits have been measured or are already disentangled to others or the oracle acts on a subspace of $(n-t)$ qubits).

- $c=\operatorname{ceil}\left(\log _{2} q\right)$, that is the least integer greater or equal to $\log _{2} q$.

- $m \leq q$ is the number of values $x$ for which $f(x)=1$.

- $r=\operatorname{int}\left(\log _{2} m\right)$ is the integer part of $\log _{2} m$.

\section{Database: signs and symptoms of the four tropical diseases}

\subsection{Short description of the four diseases}

Based on $[24,25,26,27]$ we are going to give a briefly description of the four tropical diseases of our study. Appendix A gives their signs and symptoms.

Malaria is a life-threatening disease caused by protozoan parasites of the genus Plasmodium. The parasite is generally transmitted from one human to another through the bite of infected females Anopheles mosquitoes. Five species infect humans by entering the bloodstream: P. knowlesi, P. ovale, P. malariae, P. vivax and $P$. falciparum. The last two ones, $P$. vivax and $P$. falciparum, are the greatest threat because they affect a greater proportion of the red blood cells than the others. P. falciparum is the most prevalent malaria parasite on the African continent. It is generally responsible for most malaria-related deaths. $P$. vivax has a wider distribution than P. falciparum, and predominates in many countries outside Africa. About 3.2 billion people, almost half of the world population, are exposed to malaria. Sub-saharan Africa carries a disproportionately high share of the global malaria burden. In 2015, the region was home of $89 \%$ of malaria cases and $91 \%$ of malaria deaths.

Typhoid fever, also known as salmonellosis, is a life-threatening illness caused by the bacterium Sallmonella typhi, also known as Salmonella enterica serotype typhi, growing in the intestines and blood. Dirt (poor sanitation and poor hygiene) is the main cause of transmission of the disease. Contaminated food and unsafe water are the main vectors. Typhoid fever remains a serious worldwide threat, especially in developing countries. The estimated cases of this disease are nearby 16-33 millions each year; this leads to more than half million death cases. The disease is endemic in India, Southeast Asia, Africa, South America and many other areas.

Yellow fever is an acute viral hemorrhagic disease caused by a Flavivirus. The virus is transmitted through the bite of an infected female mosquito (Aedes aegypti). The virus causes deterioration of the liver. There are an estimated 200,000 cases of yellow fever, causing 30,000 worldwide deaths each year, with $90 \%$ occurring in Africa. Forty-four endemic countries in Africa and Latin America, with a combined population of over 
900 millions, are at risk. In Africa, an estimated 508 millions people live in 31 countries exposed to the disease. The remaining population at risk are in 13 countries in Latin America. Bolivia, Brazil, Colombia, Ecuador and Peru are at greatest risk.

Dengue fever is a painful, debilitating mosquito-borne tropical disease caused by dengue virus. The virus is transmitted by several species of mosquito within the genus Aedes, principally Aedes aegypti. WHO has classified dengue as one of the neglected tropical diseases and reported the resurgence of the disease [28]. Dengue is common in more than 110 countries. It infects 50 to 528 millions people worldwide a year, leading to half million hospitalisations, and approximately 25,000 deaths. Cases of the disease have been reported at least in 22 countries in Africa; but it is likely present in all of them with $20 \%$ of the population at risk. This makes it one of the most common vector-borne diseases worldwide.

\subsection{Description of the database}

Our database contains signs and symptoms of four tropical diseases: malaria, typhoid fever, dengue fever and yellow fever (see Appendix A). There are seventeen signs and symptoms specific to malaria, eighteen to typhoid fever, twenty-three to dengue and seventeen to yellow fever. Some signs and symptoms are common to different diseases, not only the four, such as fever and headache for example, thereby our database contains ninety-five signs and symptoms. We need six qubits for the computation because each symptom is labelled with a number in its binary form. Therefore we can compute $2^{6}$ signs and symptoms per disease. In the subspace of disease we label the signs and symptoms in decimal form by starting with 0 . Furthermore, we need four qubits to label the ten groups of diseases presented by the Tab. 2 that are used in the linear QAM algorithm. There is one group per individual disease ( $\left.\mathrm{N}^{\circ} 1-4\right)$; two groups corresponding to common signs and symptoms to malaria and typhoid fever or yellow fever $\left(\mathrm{N}^{\circ} 5-6\right)$; two groups corresponding to common signs and symptoms to yellow fever and typhoid fever or dengue ( $\left.\mathrm{N}^{\circ} 7-8\right)$; one group corresponding to common signs and symptoms to malaria, yellow fever and dengue $\left(\mathrm{N}^{\circ} 9\right)$; and finally one group corresponding to common signs and symptoms to each of the four diseases. The last can be found in other diseases such as headache, fever and abdominal pain ( $\left.\mathrm{N}^{\circ} 10\right)$. For the non-linear QAM algorithm we need four flag qubits, one for each disease. It appears that we need a register that contains $n=14$ qubits, six for signs and symptoms, four for diseases for each QAM algorithm. So, there are two output registers. The labels of the output qubits for the linear QAM algorithm are mentioned above. The other possibilities are pointed to be other diseases and signs and symptoms in our model. Two sets of four qubits for output are needed due to the fact that the learning process of the linear QAM algorithm is different from the one used for the non-linear QAM algorithm. For the non-linear QAM algorithm each flag qubit is associated to one disease. That qubit allows to know if a symptom specific to a particular disease is present. Therefore value 0 means that this particular disease is not present, whereas value 1 means that it is present. Nevertheless, if the values of that four qubits are 0 , it means that none specific symptom is introduced.

\begin{tabular}{lll}
\hline $\mathbf{N}^{\circ}$ & Group of diseases by signs and symptoms & Label \\
\hline 1 & Malaria & $|0001\rangle$ \\
2 & Typhoid fever & $|0010\rangle$ \\
3 & Yellow fever & $|0100\rangle$ \\
4 & Dengue & $|1000\rangle$ \\
5 & Malaria + Typhoid fever & $|0011\rangle$ \\
6 & Malaria + Yellow fever & $|0101\rangle$ \\
7 & Yellow fever + Typhoid fever & $|0110\rangle$ \\
8 & Yellow fever + Dengue & $|1100\rangle$ \\
9 & Malaria + Yellow fever + Dengue & $|1101\rangle$ \\
10 & Other diseases & $|1111\rangle$ \\
\hline
\end{tabular}

Table 2: Groups of diseases by signs and symptoms and their labels in binary form for the linear algorithm. The hamming distance between the label of two groups of diseases is equal to 1 when the signs and symptoms are common to these two groups of diseases and is equal to 2 otherwise. The group $\mathrm{N}^{\circ} 10$ or Other diseases is devoted to signs and symptoms that are common to each 4 diseases and that can also occur in other groups of diseases not mentioned here. We point out the fact that other labels are also consider to be Other diseases.

For the linear QAM retrieving algorithm, the determination of the number of iterations is mentioned above. Labelling all the fourteen qubits from $\left|q_{1}\right\rangle$ to $\left|q_{14}\right\rangle$, the entire QAM looks as illustrated in Fig. 2. 


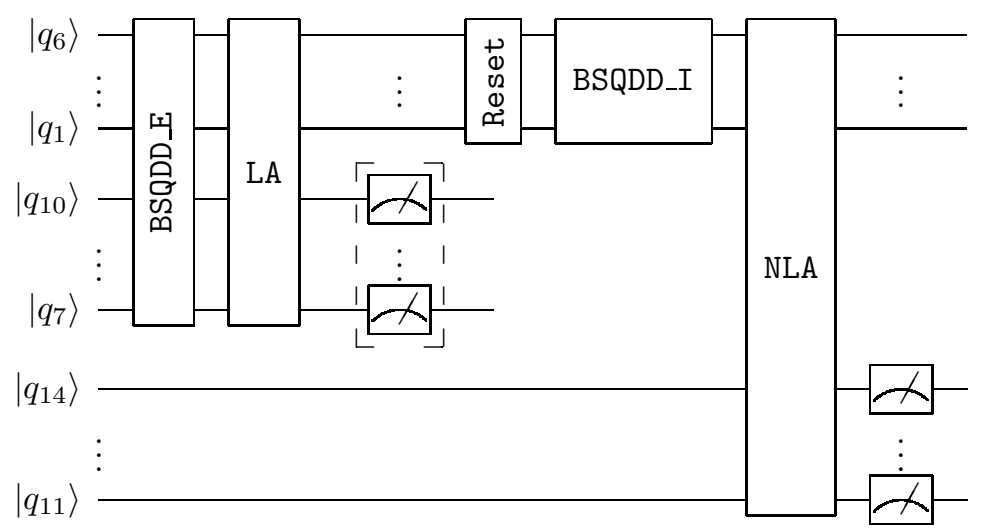

Figure 2: Schematic structure of the QAMDiagnos. BSQDDE is the learning part of the linear QAM algorithm, whereas, after reinitialisation, BSQDD_I is the learning part of the non-linear QAM algorithm. LA is the set of gates simulating the linear QAM algorithm. NLA is a repeated non-linear QAM algorithm, once for each disease. Each flag qubit from $\left|q_{11}\right\rangle$ to $\left|q_{14}\right\rangle$ is devoted to one disease.

\section{Simulations results and discussion}

To design the linear part of the QAMDiagnos, we take into account the four following facts:

1. The arbitrary value that regulates the width distribution around the chosen centre is $a=0.4999$;

2. The centre of the query is $|0000\rangle$ because we assume that there is no indication about disease;

3. The difference between the number of iterations $\Lambda$ and the nearest integer must be less than 0.1 ;

4. Not more than six signs and symptoms can be chosen.

We give in the next Tabs. 3 and 4 the average probabilities of correct recognition $\left(P_{c}\right)$. We take the average due to the convention we chose to label signs and symptoms, and diseases. Therefore, sensibility is the conditional probability to have a correct recognition if the disease is present. So, as we can obtain from our QAM probabilities of good recognition, we can see that they look like sensibilities.

\section{- For single infection}

We obtain sensibilities according to the definition.

\begin{tabular}{|c|c|c|c|c|c|c|c|c|c|c|c|}
\hline \multirow[b]{2}{*}{ Disease } & \multicolumn{6}{|c|}{ Number of signs and symptoms } & & \multirow[b]{2}{*}{$q_{11}$} & \multirow[b]{2}{*}{$q_{12}$} & \multirow[b]{2}{*}{$q_{13}$} & \multirow[b]{2}{*}{$q_{14}$} \\
\hline & 1 & 2 & 3 & 4 & 5 & 6 & & & & & \\
\hline Malaria & 93.8911 & 94.5059 & 97.5402 & 96.4843 & 99.9043 & 96.5960 & Without & 1 & 0 & 0 & 0 \\
\hline Typhoid fever & 93.8911 & 94.5059 & 97.5402 & 96.4843 & 99.9043 & 96.5960 & other & 0 & 1 & 0 & 0 \\
\hline Yellow fever & 93.8911 & 94.5059 & 97.5402 & 96.4843 & 99.9043 & 96.5960 & & 0 & 0 & 1 & 0 \\
\hline Dengue & 93.8904 & 94.5052 & 97.5397 & 96.4837 & 99.9041 & 96.5954 & signs and symptoms & 0 & 0 & 0 & 1 \\
\hline Malaria & 47.4418 & 65.0912 & 72.4296 & 79.9350 & 80.5407 & - & With 1 & 1 & 0 & 0 & 0 \\
\hline Typhoid fever & 47.4418 & 65.0912 & 72.4296 & 79.9350 & 80.5407 & - & other & 0 & 1 & 0 & 0 \\
\hline Yellow fever & 47.4418 & 65.0912 & 72.4296 & 79.9350 & 80.5407 & - & & 0 & 0 & 1 & 0 \\
\hline Dengue & 47.4411 & 65.0905 & 72.4289 & 79.9346 & 80.5400 & - & sign or symptom & 0 & 0 & 0 & 1 \\
\hline Malaria & 32.6307 & 48.3689 & 59.9605 & 64.4826 & - & - & With 2 & 1 & 0 & 0 & 0 \\
\hline Typhoid fever & 32.6307 & 48.3689 & 59.9605 & 64.4826 & - & - & other & 0 & 1 & 0 & 0 \\
\hline Yellow fever & 32.6307 & 48.3689 & 59.9605 & 64.4826 & - & - & & 0 & 0 & 1 & 0 \\
\hline Dengue & 32.6302 & 48.3683 & 59.9600 & 64.4819 & - & - & signs and symptoms & 0 & 0 & 0 & 1 \\
\hline Malaria & 24.3022 & 39.9808 & 48.4219 & - & - & - & With 3 & 1 & 0 & 0 & 0 \\
\hline Typhoid fever & 24.3022 & 39.9808 & 48.4219 & - & - & - & other & 0 & 1 & 0 & 0 \\
\hline Yellow fever & 24.3022 & 39.9808 & 48.4219 & - & - & - & & 0 & 0 & 1 & 0 \\
\hline Dengue & 24.3018 & 39.9803 & 48.4213 & - & - & - & signs and symptoms & 0 & 0 & 0 & 1 \\
\hline
\end{tabular}

Table 3: Average probabilities $P_{c}$ according to the number of signs and symptoms related to a disease in case of a single infection for the linear part of the QAM. For the non-linear part, each corresponding flag qubit has its value equal to 1 . Here "other signs and symptoms" are fever, headache and abdominal pain. 


\section{- For polyinfection}

We also obtain sensibilities according to the definition.

\begin{tabular}{lccccl}
\hline & \multicolumn{2}{c}{ Number of symptom of malaria } & \\
\cline { 2 - 5 } Disease & $\mathbf{0}$ & $\mathbf{1}$ & $\mathbf{2}$ & $\mathbf{3}$ & \\
\hline Malaria & 0.1577 & 24.2913 & 39.9633 & 48.4079 & With 1 sign \\
Typhoid fever & 32.6167 & 24.2913 & 19.9844 & 16.2842 & or symptom \\
Malaria + Typhoid f. & 65.0886 & 48.3653 & 39.9685 & 32.3538 & of Typhoid f. \\
\hline Malaria & 0.2283 & 19.9844 & 32.3460 & - & With 2 signs \\
Typhoid fever & 48.3543 & 39.9633 & 32.3460 & - & and symptoms \\
Malaria + Typhoid f. & 48.3653 & 39.9685 & 32.3538 & - & of Typhoid f. \\
\hline Malaria & 0.0055 & 16.2842 & - & - & With 3 signs \\
Typhoid fever & 59.9422 & 48.4079 & - & - & and symptoms \\
Malaria + Typhoid f. & 39.9685 & 32.3538 & - & - & of Typhoid f. \\
\hline Malaria & 0.2224 & - & - & - & With 4 signs \\
Typhoid fever & 64.4697 & - & - & - & and symptoms \\
Malaria + Typhoid f. & 32.3538 & - & - & - & of Typhoid f. \\
\hline \hline$q_{11}$ & 0 & 1 & 1 & 1 & \\
$q_{12}$ & 1 & 1 & 1 & 1 & \\
$q_{13}$ & 0 & 0 & 0 & 0 & \\
$q_{14}$ & 0 & 0 & 0 & 0 & \\
\hline
\end{tabular}

Table 4: Probabilities $P_{c}$ according to the number of signs and symptoms related to diseases in case of a polyinfection for the linear part of the QAM. For the non-linear part, each corresponding flag qubit has its value equal to 1 . Here "common sign or symptom" is the one that is common to the two diseases. Here we have chosen two common signs and symptoms.

As we can see on the Tabs. 3 and 4 the QAM can collapse to a state representing single infection or polyinfection. That is the QAM can distinguish single from polyinfection. That distinction is possible with the lowest (one) or the highest (six) number of particular signs and symptoms of a disease, but it is better in the last case. When the "other signs and symptoms" are inserted, the QAM can also do the distinction. As we observe on the Tab. 5 the "other signs and symptoms" are not related to a particular disease. Therefore, the non-linear part of the QAM completes or corrects the results of the linear part.

\begin{tabular}{lccc}
\hline & \multicolumn{3}{c}{ Number of signs and symptoms } \\
\cline { 2 - 4 } Disease & $\mathbf{1}$ & $\mathbf{2}$ & $\mathbf{3}$ \\
\hline Other diseases & 96.38 & 96.73 & 98.53 \\
\hline
\end{tabular}

Table 5: Probabilities $P_{c}$ according to the number of "other signs and symptoms" for linear part of the QAM. For the non-linear part, each flag qubit has its value equal to 0. The QAM does not associate these signs and symptoms to one of the four diseases.

We also evaluate the specificity of the QAM. Specificity is the conditional probability to have a correct recognition if the disease is not present. In other words it is the ability of the memory to distinguish healthy people from non-healthy ones. For our QAM, to acquire it we use the 448 signs and symptoms without any relation with the four diseases and take the average of probabilities to obtain "Other diseases" as a result. We obtain the Tab. 5 and assume that the specificity is $\mathbf{9 6 . 3 8} \%$.

\section{QAMDiagnos: desktop and smart-phone GUI}

All the simulations and results were made by encoding the algorithms in C++ language. A multi-platform friendly graphical user interface (GUI) of our QAMDiagnos is designed for the medical staff. (see figures of supplementay material for more details). It is developed with the open source version of the C++ library Qt5.

To use the software, after observing or discussing with a patient, and according to what he observes and the answers of the patient, the physician introduces signs and symptoms in the QAMDiagnos (at least one sign or symptom and a maximum of six signs and symptoms). The result and a proposal of treatment are given in the text box "Treatment proposal" for the desktop GUI or in the only one text box for the smart-phone GUI. Although weight and age can be important for accurate diagnosis, the QAMDiagnos does not use these data because it can occur that the physician forgets to take them or not. Therefore, in the text box "Treatment proposal" the 
QAMDiagnos shows a treatment proposal which is not bound to the weight or age. We made the simulations on a classical computer, so we can obtain each probability (recognition efficiency).

Thus, the physician can also compare the recognition efficiency of the linear part of the QAMDiagnos for each disease given in the "Results" area. So, the disease with the greatest recognition efficiency can be viewed as the corresponding disease of the patient. To achieve this goal, that is to simulate the probabilistic nature of the quantum theory, we use the qrand() function of Qt5 that allows us to choose arbitrarily one of the diseases according to its probability. As the linear QAM algorithm is the main algorithm, the disease with the highest probability is given as result with the associated treatment proposal. Before that, the non-linear QAM algorithm identifies if a sign or symptom specific to a particular disease is present. The results of the linear QAM algorithm and the non-linear QAM algorithm are given together to help the physician in his decision.

The complementary of the two algorithms is more highlighted in the case of polyinfection. For example, if common signs and symptoms to malaria, yellow fever and dengue are inserted and symptom particular to malaria and another to yellow fever are also inserted, the linear QAM algorithm will identify this combination as a polyinfection. But, the non-linear QAM algorithm will identify the signs and symptoms of malaria and yellow fever. This gives to the physician the possibility to focus only on two diseases (malaria and yellow fever) instead of the three.

Some snapshots of the QAMDiagnos allowing better understanding are available as supplementary material associated to this paper.

\section{Conclusion}

We have presented in this paper the QAMDiagnos, a Quantum Associative Memory framework that can be helpful to diagnose four tropical diseases (malaria, typhoid fever, yellow fever and dengue) with several common signs and symptoms. The framework that has a friendly multi-platform GUI, is a combination of the improved versions of original quantum linear retrieving algorithm proposed by Ventura and the non-linear quantum search algorithm of Abrams and Lloyd. While the phase-inversion introduced in the original linear QAM algorithm increases the capacity of the memory to make a good diagnosis, the non-linear QAM algorithm helps to confirm or to correct the diagnosis and to make some suggestions to the medical staff for the treatment. In addition, the QAMDiagnos can distinguish a single infection from a co-infection and needs only few specific signs and symptoms of disease and common signs and symptoms. Therefore, the QAMDiagnos framework can be a good tool to help inexperienced medical staff or medical staff without laboratory facilities to rapidly and accurately diagnose malaria, typhoid fever, yellow fever and dengue, four tropical diseases that are often confused. Due to its highly automated nature, health centres personnel can be trained to operate the QAMDiagnos in just one day.

For future works, it is planned to build up a device that can help acquire physiological parameters on a patient and transfer them to the QAMDiagnos.

\section{Acknowledgements}

We thank Pr. Paul WOAFO for his helpful discussions and remarks. We also thank Dr. OKALA, Dr. DOUALLA and Pr. LUMA of the Hopital Général de Douala (Cameroon) for their remarks and their help on the classification of signs and symptoms. We also thank Moise SOH, the Senior Translator, for proofreading our work.

\section{References}

[1] Richard Dybowski and Vanya Gant, editors. Clinical Applications of Artificial Neural Networks. Cambridge University Press, december 2007.

[2] Amato Filippo, López Alberto, Peña-Méndez Eladia María, Vaňhara Petr, Hampl Aleš, and Havel Josef. Artificial neural networks in medical diagnosis. Journal of Applied Biomedicine, 11:47, 2013.

[3] Erdem Alkim, Emre Gürbüz, and Erdal Kiliç. A fast and adaptive automated disease diagnosis method with an innovative neural network model. Neural Networks, 33:88-96, 2012.

[4] Aldape-Pérez Mario, Yáñez-Márquez Cornelio, Camacho-Nieto Oscar, and J.Argüelles-Cruz Amadeo. An associative memory approach to medical decision support systems. Comput Methods Programs Biomed, 106(3):287-307, jun 2012.

[5] J. J. Hopfield. Neural Networks and Physical Systems with Emergent Collective Computational Abilities. Proceedings of the National Academy of Sciences, 79:2554-2558, 1982. 
[6] J. J. Hopfield. Neurons with Graded Response Have Collective Computational Properties Like Those of Two-state Neurons. Proceedings of the National Academy of Sciences, 81:3088-3092, 1984.

[7] A.Sunny Ochi-Okorie. Combining medical records with case-based reasoning in a mixed paradigm design TROPIX architecture \& implementation. In DavidB. Leake and Enric Plaza, editors, Case-Based Reasoning Research and Development, volume 1266 of Lecture Notes in Computer Science, pages 94-103. Springer Berlin Heidelberg, 1997.

[8] Neetu Ahirwar, Sapnojit Pattnaik, and Bibhudendra Acharya. Advanced image analysis based system for automatic detection and classification of malarial parasite in blood images. International Journal of Information Technology and Knowledge Management, 5(1):59-64, January-June 2012.

[9] Khalda F. Ali, Amir Mohamed Elamir, and Riza. M. Suliman. Article: A Comparative Study of Three Intelligent Techniques for Malaria in Africa Continent. International Journal of Computer Applications, 67(2):1-5, April 2013. Published by Foundation of Computer Science, New York, USA.

[10] Bruno Andrade, Antonio Reis-Filho, Austeclino Barros, Sebastiao Souza-Neto, Lucas Nogueira, Kiyoshi Fukutani, Erney Camargo, Luis Camargo, Aldina Barral, Angelo Duarte, and Manoel Barral-Netto. Towards a precise test for malaria diagnosis in the Brazilian Amazon: comparison among field microscopy, a rapid diagnostic test, nested PCR, and a computational expert system based on artificial neural networks. Malaria Journal, 9(1):117, 2010.

[11] World Malaria Report. Technical report, World Health Organisation, 2012.

[12] J.-P. Tchapet Njafa, S.G. Nana Engo, and Paul Woafo. Quantum Associative Memory with Improved Distributed Queries. International Journal of Theoretical Physics, 52(6):1787-1801, June 2013.

[13] J.-P. Tchapet Njafa and S.G. Nana Engo. Concise quantum associative memories with non-linear search algorithm. Fortschr. Phys., 64(2-3):250-268, February 2016.

[14] A.M.Agarkar and Dr. A.A.Ghatol. FFANN Based Cost Effective Major Infant Disease Management. International Journal of Computer Applications, 7(11):29-33, October 2010. Published By Foundation of Computer Science.

[15] A. A. Ezhov, A. V. Nifanova, and Dan Ventura. Quantum associative memory with distributed queries. Inf. Sci. Inf. Comput. Sci., 128(3-4):271-293, October 2000.

[16] D. Ventura and T.R. Martinez. Quantum Associative Memory. Inf. Sci. Inf. Comput. Sci., 124(1-4):273-296, 2000 .

[17] Michaël Defoin Platel, Stefan Schliebs, and Nikola Kasabov. Quantum-inspired Evolutionary Algorithm: A Multimodel EDA. Trans. Evol. Comp, 13(6):1218-1232, December 2009.

[18] Stefan Schliebs, Michaël Defoin-Platel, Sue Worner, and Nikola Kasabov. Integrated feature and parameter optimization for an evolving spiking neural network: Exploring heterogeneous probabilistic models. Neural Networks, 22(5-6):623-632, 2009. Advances in Neural Networks Research: \{IJCNN20092009\} International Joint Conference on Neural Networks.

[19] Stefan Schliebs, Nikola Kasabov, and Michael Defoin-Platel. On the Probabilistic Optimization of Spiking Neural Networks. Int. J. Neural Syst., 20(6):481-500, 2010.

[20] Nikola Kasabov. To spike or not to spike: A probabilistic spiking neuron model. Neural Networks, 23(1):16$19,2010$.

[21] David Rosenbaum. Binary superposed quantum decision diagrams. Quantum Information Processing, 9(4):463-496, August 2010.

[22] D. Ventura and T.R. Martinez. Initializing the Amplitude Distribution of a Quantum State. Foundations of Physics Letters, 6:547-559, December 1999.

[23] Daniel S. Abrams and Seth Lloyd. Nonlinear Quantum Mechanics Implies Polynomial-Time Solution for NP-Complete and \# P Problems. Phys. Rev. Lett., 81:3992-3995, Nov 1998.

[24] E. Pilly. Maladies infectieuses et tropicale. Vivactis Plus, october 2011.

[25] Jeremy Farrar et al. Manson's Tropical Infectious Diseases. Saunders Ltd, 23 edition, 2013. 
[26] CMIT. ECN.PILLY, Maladies infectieuses et tropicales. Alinéa Plus, 4e edition, 2016.

[27] Guide clinique et thérapeutique. Médécins Sans Frontières, August 2016.

[28] Maladies tropicales négligées. Technical report, World Health Organisation, 2010.

\section{A Symptoms of each group of diseases}

\begin{tabular}{|c|c|c|c|}
\hline Malaria & Typhoid fever & Yellow fever & Dengue \\
\hline Headache & Headache & Headache & Headache \\
\hline Abdominal pain & Abdominal pain & Abdominal pain & Abdominal pain \\
\hline Fever & Fever & Fever & Fever \\
\hline Myalgia & & $\overline{\text { Myalgie }}$ & Myalgia \\
\hline Renal failure & & Renal failure & Renal failure \\
\hline Nausea & & Nausea & Nausea \\
\hline Vomiting & & Vomiting & Vomiting \\
\hline Shiver or cold sensation & & Shiver or cold sensation & \\
\hline Hemorrhage & & Hemorrhage & \\
\hline Iterus & & Iterus & \\
\hline Oliguria & & Oliguria & \\
\hline Anorexia & Anorexia & & \\
\hline Coma & Coma & & \\
\hline \multirow[t]{8}{*}{ Prostration } & Prostration & & \\
\hline & Epistaxis & Epistaxis & \\
\hline & Myocarditis & Myocarditis & \\
\hline & & Rachiodynia & Rachiodynia \\
\hline & & Heart failure & Heart failure \\
\hline & & Hepatic failure & Hepatic failure \\
\hline & & Conjunctival injection & Conjunctival injection \\
\hline & & Somnolence & Somnolence \\
\hline Anemia & Asthenia & Acidosis & $\begin{array}{l}\text { Accumulation of fluid and } \\
\text { respiratory distress }\end{array}$ \\
\hline Shock & Bouveret's ulcer & $\begin{array}{l}\text { Bradycardia related to tem- } \\
\text { perature }\end{array}$ & Generalized adenopathy \\
\hline $\begin{array}{l}\text { Generalized or focal convul- } \\
\text { sion }\end{array}$ & Relative bradycardia & Infectious shock & Agitation or Lethargy \\
\hline Multiple convulsion & Constipation & Terminal coma & Arthralgia \\
\hline Stiffeness & Yellowy diarrhea & Excessive dryness & Asthnie prolonge \\
\hline Delirium & Encephalitis & Back pain & $\begin{array}{l}\text { Elevation of the hematocrit } \\
\text { and fast drop of platelets }\end{array}$ \\
\hline Respiratory distress & $\begin{array}{l}\text { Lenticular exanthem of limbless } \\
\text { man }\end{array}$ & Limb pain & Corrected shock \\
\hline Diarrhea & Rumble in the right iliac cavity & Renal pain & Uncorrected shock \\
\hline Hemoglobinuria & $\begin{array}{l}\text { Digestive hemorrage or digestive } \\
\text { perforation }\end{array}$ & Epigastric pain & Tensor fall \\
\hline Hepatosplenomegaly & Insomnia & Fatigue & $\begin{array}{l}\text { Desquamation of macular } \\
\text { eruption }\end{array}$ \\
\hline Hypoglycemia & Sabural tongue & Foul breath & Retro-orbital pain \\
\hline Malaise & Abdominal heavines & Hypotension & Encephalopathy \\
\hline Pulmonary edema & Peritonitis & Lumbago & Macular eruption \\
\hline Palenes & Dissociate pulse & $\begin{array}{l}\text { Prolongation of the PR and } \\
\text { QT intervals on electrocar- } \\
\text { diography }\end{array}$ & Maculopapular exanthem \\
\hline Sub-iterus & Septicemia & Light proteinuria & Hepatomegaly $(>2 \mathrm{~cm})$ \\
\hline Profuse sweat & Splenomegaly & Purpura & Sudden hypothermia \\
\hline \multirow[t]{6}{*}{ Conscience trouble } & Moderate splenomegaly & Vomito negro & Leukopenia \\
\hline & Typhoid state & & Obnubilation \\
\hline & & & Vascular purpura \\
\hline & & & $\begin{array}{l}\text { Benign cutaneous or mucous } \\
\text { bleeding }\end{array}$ \\
\hline & & & Severe cutaneous or mucous \\
\hline & & & Persitent vomiting \\
\hline
\end{tabular}

Table 6: signs and symptoms of the database [24, 25, 26, 27]. 